\title{
DISEÑO DE UN MODELO DE DESARROLLO COOPERATIVISTA EN ECONOMÍAS POPULARES Y SOLIDARIAS, BASADO EN LA EXPERIENCIA DE LA COOPERATIVA EL SALINERITO DE LA CIUDAD DE GUARANDA
}

\author{
DESIGN OF A MODEL OF COOPERATIVE DEVELOPMENT IN POPULAR AND \\ SOLIDARITY ECONOMY, BASED ON THE EXPERIENCE OF THE COOPERATIVA \\ EL SALINERITO FROM \\ GUARANDA
}

EDGAR DANIEL JIMÉNEZ-BONILLA

Universidad Católica de Santiago de Guayaquil. edgar.jimenez@cu.ucsg.edu.ec

RESUMEN

Se considera al cooperativismo como un proceso dinámico que une a la comunidad a través de un modelo de desarrollo empresarial para satisfacer las cambiantes necesidades materiales e inmateriales de los socios con los beneficios generados en el mismo. El caso "Cooperativa El Salinerito" Se propone un proceso que es modelado en un diagrama con cinco factores: motivantes, liderazgo, recursos, tipo de gobierno y beneficios esperados. El cual sirve como una herramienta que ayuda a las cooperativas, socios y gobiernos a evaluar su situación actual y plantear estrategias de crecimiento, así como un mapa de planificación estratégica para crear nuevos emprendimientos cooperativistas.

PaLABRAS CLAVE: cooperativismo, modelo, emprendimiento, economía popular y solidaria.
ABSTRACT

It is considered the cooperativism as a dynamic process that joins the coaammunity through a business development model to satisfy the partners's changing materials and immaterials needs with profits generated in the process. From the study case "Cooperativa El Salinerito" this process is exemplified with a diagram that involves five factors: motivational, leadership, resources, type of government and expected benefits, which serves as a tool to help cooperativism, partners and governments to assess their current situation and suggest strategies for growth; as well as a map of strategic planning to create new cooperative ventures.

KEYWORDS: cooperativis, model, entrepreneurship, popular and solidarity economics. 
INTRODUCCIÓN

En la actualidad el Gobierno Nacional se encuentra impulsando la creación y desarrollo de Economías Populares y Solidarias (EPS) a través de leyes y líneas de financiamiento. A pesar de esta iniciativa, se ha detectado que no existe una metodología para planificar ni administrar la creación de cooperativas que impulsen este tipo de economías. Por ello, se evidencia la necesidad de contar con un modelo que sirva de base para definir y planear estrategias en organizaciones de esta naturaleza.

El desarrollo de un modelo de negocio es un concepto en donde se puede exponer de forma holística el funcionamiento de una empresa, a través de la interacción de módulos o partes que explican las acciones, recursos y asociaciones tanto internas como externas del negocio (Osterwalder y Pigneur, 2012). No obstante, el mismo aún no se encuentra definido para el caso de las EPS.

El desarrollo de estas economías implica la generación de miles de fuentes de trabajo en el país (Danani, 2004), que principalmente buscan mejorar la calidad de vida de la comunidad participante. Sin embargo, el índice de fracaso en el desarrollo de estos modelos de negocio es muy alto debido a la falta de formatos estructurados para la creación y desarrollo de los mismos.

Es imperativo entonces, explicar y esquematizar la creación, el desarrollo y funcionamiento de estas economías que actualmente se encuentran en auge y que gozan del total apoyo de gobiernos a través de modelos de inclusión social y mejoramiento de la calidad de vida de las comunidades (Laville, 2004).

Un modelo de desarrollo cooperativista adaptado a la realidad de las EPS es una herramienta que permitirá planificar, organizar, dirigir y controlar la creación de empresas y comunidades de economía popular y solidaria, potenciando así su crecimiento y mejorando la calidad de vida de las personas que se involucren en la misma.

Por esta razón el presente estudio tiene como objetivo principal esquematizar el desarrollo cooperativista de la comunidad de Salinas de Guaranda, el cual servirá como línea de base para la formulación de un modelo de desarrollo para este tipo de organizaciones.

ANTECEDENTES

En Ecuador las cooperativas están supervisadas y controladas por la Superintendencia de
Economía Popular y Solidaria (SEPS) de acuerdo a la Ley Orgánica de Economía Popular y Solidaria del Sistema Financiero (Asamblea Nacional de la República del Ecuador, 2011). Estas se encuentran distribuidas, como se muestran en la Tabla 1, en: 899 financieras y 2,402 no financieras, más 113 en estado de liquidación.

ANÁLISIS DE LOS RESULTADOS

TABLA 1. SECTOR COOPERATIVO DEL ECUADOR

\begin{tabular}{|c|c|c|c|}
\hline \multicolumn{4}{|c|}{ NÚMERO DE COOPERATIVAS DEL ECUADOR } \\
\hline ESTAdO & $\begin{array}{c}\text { NO } \\
\text { FINANCIERAS }\end{array}$ & $\begin{array}{l}\text { DE AHORRO Y } \\
\text { CREEDITO }\end{array}$ & TOTAL \\
\hline Activa & 2402 & 897 & 3299 \\
\hline Intervenidas & 0 & 2 & 2 \\
\hline Total & 2402 & 899 & 3301 \\
\hline
\end{tabular}

Fuente: El autor.

Las cooperativas financieras se refieren a las Cooperativas de Ahorro y Crédito, las cuales por su naturaleza, además son supervisadas y reguladas por la Superintendencia de Bancos. Las No Financieras son aquellas cooperativas de vivienda, construcción, producción, de transporte público, entre otras. Estas son controladas únicamente por la SEPS.

El presente estudio se sustenta principalmente en la necesidad de desarrollar métodos de planificación estratégica para las 2,402 cooperativas no financieras en estado activo que actualmente existen en el Ecuador. Estas cooperativas están concentradas principalmente en las provincias de Guayas, Manabí, Azuay, Tungurahua y Pichincha denotadas con el color azul en la Figura 1.

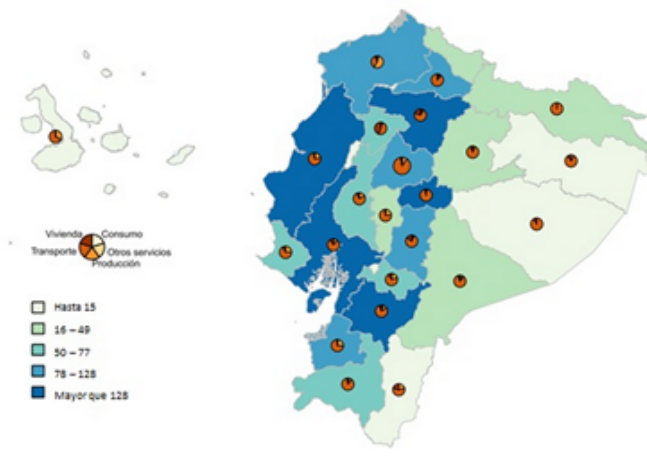

Figura 1. Distribución de cooperativas no financieras por tipo

FORMULACIÓN DEL PROBLEMA DE INVESTIGACIÓN

En el Ecuador existen muchas comunidades con limitado acceso a los servicios de salubridad, educación y vías de comunicación; situación que dificulta el desarrollo económico de las mis- 
mas. Sin embargo, el gobierno actualmente se encuentra impulsando el desarrollo de las EPS a través de leyes y líneas de financiamiento para el sector (SENPLADES, 2009).

El objetivo de la medida mencionada, es mejorar la calidad de vida de las personas, a través de la generación de empleos en donde se anteponga la vida de los seres humanos sobre el capital (Coraggio, 2004a). A pesar de este impulso, no se evidencia una metodología clara para la creación y desarrollo de un modelo de negocio de EPS en el país, generando un gran número de problemas en la planificación e implementación de los proyectos y reduciendo la probabilidad de éxito de los emprendimientos.

La creación de un modelo aplicado a empresas que se desarrollan en EPS será entonces una herramienta para planificar y aplicar en gran parte de los proyectos que apuntan al desarrollo de comunidades marginales a través del desarrollo económico de las mismas. Además, el modelo puede ser aplicable a entornos personales sirviendo de herramienta para el planteamiento de objetivos familiares para las personas que trabajan en EPS. Es relevante entonces, conocer ¿Qué factores económicos y sociales influyen en el desarrollo de las cooperativas en Economías Populares y Solidarias?

\section{MARCO TEÓRICO}

La economía consiste en movilizar o generar, distribuir y organizar combinaciones de recursos "escasos" (Schüttz, 2011) para poder producir, intercambiar y utilizar bienes y servicios para satisfacer de la mejor manera las necesidades del ser humano. Esto ha generado conflictos dentro de la sociedad y luchas sociales, a pesar que el objetivo primordial de la economía es que nadie sufra por necesidades que materialmente son posibles de satisfacer.

Aquí nace el concepto de "Economía Popular" considerando a la misma como el conjunto de actividades económicas y prácticas sociales orientadas principalmente a los sectores más vulnerables (Coraggio, 2004a), con el objetivo de "satisfacer las necesidades básicas, tanto materiales como inmateriales de los individuos" (Sarria y Tiribia, 2004 p.173).

La EPS está orientada fundamentalmente como modelo aplicable a los más pobres (Toloza, Delgado, Chehab, Verdesoto, y Demenus, 2012), hacia aquellas personas que tienen recursos limitados y que en muchos de los casos deben desarrollar sus actividades en un ambiente y condiciones poco favorables; mermando sus ánimos de superación y alentando la transgeneracionalidad de la pobreza.

Para el Ecuador a través de La Ley Orgánica de Economía Popular y Solidaria y del Sistema Financiero Popular y Solidario la EPS es considerada como:

"La forma de organización económica, donde sus integrantes, individual o colectivamente, organizan y desarrollan procesos de producción, intercambio, comercialización, financiamiento y consumo de bienes y servicios, para satisfacer necesidades y generar ingresos, basadas en relaciones de solidaridad, cooperación y reciprocidad, privilegiando al trabajo y al ser humano como sujeto y fin de su actividad, orientada al Buen Vivir, en armonía con la naturaleza, por sobre la apropiación, el lucro y la acumulación de capital" (Superintendencia de Economía Popular y Solidaria, 2012, p.4).

Entonces, la EPS no se trata de una forma de economía caritativa o programas sociales para personas pobres que buscan dádivas, sino de "una economía con profundo sentido político, porque esta supone una redistribución del poder, y otra forma de ejercicio del mismo" (Coraggio, 2004, p.1). Además de un modelo administrativo con reglas y condiciones distintas a las que tradicionalmente se usa en los negocios comunes donde el fin es mejorar las calidad de vida de los involucrados (Migliaro, 1993) (Núñez, 1996).

Se trata de construir una ciudadanía y comunidad, garantizando a todos el acceso a los mismos derechos y a compartir las responsabilidades por la supervivencia de la sociedad, a través del desarrollo de programas que fomenten la participación activa (SENPLADES, 2009a); siendo conscientes que la cooperación entre todos es primordial para que el progreso de la sociedad sea verdaderamente un éxito y se logre hacer conciencia sobre la posibilidad de una mejor forma de vida (Yunus, 2011).

\section{MARCO LEGAL}

El presente proyecto tiene como referencia al Plan Nacional del Buen Vivir (PNBV) y la Ley de Economía Popular y Solidaria (LEPS) en cuyo primer artículo destaca a la EPS como una forma de organización económica que permite a los ciudadanos integrarse colectivamente con el fin de organizar y desarrollar "procesos 
de producción, intercambio, comercialización, financiamiento y consumo de bienes y servicios" (Superintendencia de Economía Popular y Solidaria, 2012, p.4).

Mientrasqueel PNBV busca cambiarel patrón de especialización de la economía de primario, extractivista hacia un patrón concentrado en la exportación debido a que "supone el aparecimiento de desigualdades irracionales y sujeto a una dinámica de rendimientos decrecientes a escala, en la que los incrementos en la inversión dan cada vez menores beneficios económicos" (SENPLADES, 2009, p. 60). Es así que el PNBV antepone al ser humano como sujeto y fin de la actividad, orientada al buen vivir, en armonía con la naturaleza, por sobre la apropiación, el lucro y la acumulación de capital.

Para ello se permite la conformación de organizaciones en sectores comunitarios, asociativos y cooperativistas, así como también las Unidades Económicas Populares de acuerdo al artículo 8 de la LEPS. Sustentado en el artículo 4 los principios para lograr el buen vivir y del bien común evitando "la prelación del trabajo sobre el capital y de los intereses colectivos sobre los individuales".

\section{METODOLOGÍA}

Se realizó una investigación de carácter exploratoria usando fuentes primarias y secundarias. Se utilizaron las técnicas de grupos focales y entrevistas a personas mayores de la comunidad desde el mes de noviembre 2013 a marzo 2014. Esto permitió definir el contexto en el que se creó el modelo cooperativita "El Salinerito".

Se usó de línea de base para esquematizar un modelo básico de desarrollo y luego validado con otros procesos de desarrollo cooperativista como se plantea en el proseso siguiente:

1. Levantamiento de información de la historia del proceso a través de fuentes primarias y secundarias. Se usaron las técnicas de grupos focales y entrevistas a personas mayores de la comunidad. Esto permitió definir el contexto en que se creó el modelo "El Salinerito".

2. Entrevistas en profundidad a directivos de las empresas comunitarias y áreas de soporte institucional desde el mes de noviembre de 2013 a marzo de 2014. Esto permitió definir el modelo de gestión aplicado y definir la relación entre micro-empresas, áreas de soporte administrativo, comercial y logístico.
3. Grupos focales desde el mes de noviembre de 2013 a marzo de 2014 con trabajadores de las microempresas participantes del sistema, para determinar las ventajas y desventajas del modelo.

4. Se procedió a través de la aplicación de encuestas a los trabajadores, directivos y expertos, a definir las variables que afectan sustancialmente al modelo desarrollado en Salinas de Guaranda.

5. En el levantamiento de información previo se obtuvieron datos que luego fueron tabulados para determinar la existencia de clusters en las variables encontradas y definir las partes del modelo a plantearse en este proyecto.

6. Se evaluó la aplicación del modelo en experiencias similares de economías populares y solidarias en otras comunidades del país y casos de estudio internacionales previamente documentados.

7. Se realizaron los ajustes necesarios al modelo y se estructuró la metodología de aplicación.

\section{RESULTADO DEL ESTUDIO}

La formación de cooperativas tiene por objetivo satisfacer necesidades de los miembros de una comunidad a través de procesos productivos donde participan los socios. De igual manera los miembros de las cooperativas van cambiando sus motivaciones a medida que se cubren sus necesidades básicas de acuerdo a la pirámide de necesidades de Maslow.

Este dinamismo hace que el modelo sea ideal para ayudar a las comunidades a cubrir sus necesidades. No obstante, presenta muchas limitaciones y conflictos en su gestión debido a la dificultad de adaptarse a los cambios que conlleva la competencia de la industria y del comportamiento de sus miembros.

Como principal problema se observa que los procesos cooperativistas plantean generar un mismo beneficio para los socios por un largo período de tiempo, sin percatarse que sus socios van cambiando en su comportamiento y requieren otro tipo de beneficios de forma progresiva.

Este es el caso de la cooperativa de "Salinas de Guaranda" la cual se creó con el objetivo de generar fuentes de empleo para los miembros de su comunidad. Actualmente enfrenta problemas para mantener un crecimiento adecuado y hacer frente a la competencia de la industria. De igual forma, invierte en maquinarias para 
reducir el costo de producción, lo que conllevará a no generar más fuentes de empleo para la comunidad.

En esta línea se debe decidir si se mantiene un modelo de cooperativa poco competitiva que genera un número limitado de empleos o si se convierte en una empresa con un modelo capitalista que maximice los beneficios de los socios iniciales ofreciéndoles satisfacer una nueva necesidad como el crecimiento económico que los mismos esperan.

Si se opta por el modelo de empresa capitalista se requiere cambiar su modelo de gestión y adaptarse a nuevas formas de negocio enfocándose en solucionar los problemas o satisfaciendo las necesidades de los consumidores (Osterwalder y Pigneur, 2012). Siendo esta acción la que provoque generar beneficios para la empresa y el mismo sea repartido para sus socios o accionistas.

Sin embargo, si se desea mantener un modelo enfocado a generar empleo debe tenerse claro que la cooperativa se enfrentará a dos problemas: 1.- El cambio de comportamiento de los socios fundadores que ya consiguieron empleo y estabilidad laboral pero que ahora requieren otro tipo de beneficios como el desarrollo profesional logrado en las grandes empresas; y 2.- El generar fuentes de empleo obliga a mantener muchos procesos de forma manual, incrementando los costos de producción y disminuyendo las ventajas competitivas en la industria a la que pertenece la cooperativa.

Hay que redefinir cuál es el objetivo de formar una cooperativa y entender que el objetivo inicial debe cambiar una vez que este se logra, puesto que sus miembros querrán crecer más a medida que la cooperativa lo hace. Si esto no sucede empezarán a desmotivarse y surgirán problemas entre la dirección de sus socios. Por otro lado, si se mantiene el crecimiento continuo de la empresa y se busca satisfacer las necesidades que tienen los socios a medida que el proceso cooperativista avanza entonces el proyecto se verá obligado a convertirse en una empresa que genere altos niveles de rentabilidad, mejorando el beneficio obtenido por los socios fundadores.

En sí, la cooperativa es una empresa que se crea para satisfacer las necesidades de sus fundadores. Entonces, por qué no tratarla como tal y entender que su crecimiento natural conlleva a convertirse en una empresa similar a las de mercado pero con un número de accionistas mucho más grande según sea el grupo de socios fundadores.
Por estas razones, definimos al cooperativismo como un proceso que transforma una comunidad dispersa en un modelo de desarrollo empresarial cuyo objetivo es satisfacer las necesidades que motivan su unión. Este proceso se grafica en la Figura 2, donde se explica, que es la comunidad la que se motiva a asociarse para obtener un beneficio a través de un modelo empresarial.

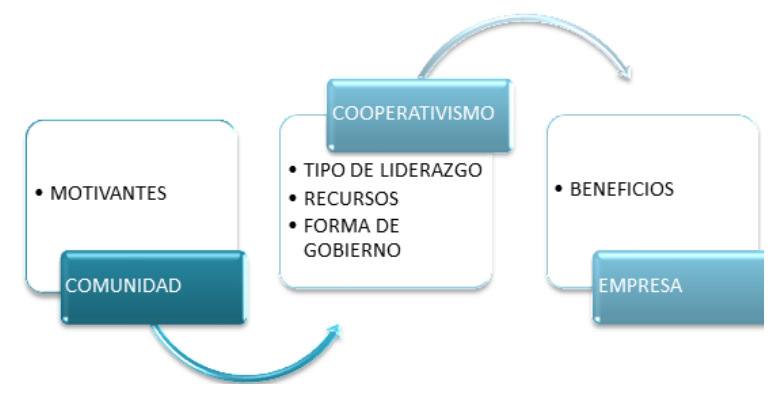

Figura 2. Proceso cooperativista

Este proceso incorpora las tres etapas fundamentales del cooperativismo: 1.- Motivantes que la comunidad tiene para asociarse; 2.- La organización cooperativista formada con algún tipo de liderazgo, obtención de recursos y la definición de un tipo de gobierno que administra y dirige el proceso; y 3.- El beneficio esperado por los miembros de la comunidad a través de un modelo de desarrollo empresarial.

Para ejemplificar el proceso, se puede tomar a un grupo de recicladores informales que requieren mejorar sus ingresos. Este grupo entonces representa una comunidad y se plantea un modelo de cooperativismo para ayudar a satisfacer esta necesidad de acuerdo a un proyecto presentado por Ormaza (2013). La formación de esta cooperativa requiere del liderazgo de una persona capaz de unir a este grupo que tiene un bajo nivel de escolaridad y prácticamente vive en la indigencia. A partir de aquí, se plantea un tipo de liderazgo religioso que los motive a asociarse en torno a la fe de conseguir días mejores para sus familias.

Una vez que los mismos se asocien por sus condiciones solo podrán aportar con mano de obra para que el proyecto funcione, es cuando el liderazgo religioso puede motivar y trabajar en la consecución de donaciones para obtener los recursos adicionales que el proyecto requiere. Finalmente, se necesita formar una directiva que administre los recursos y organice el trabajo entre los socios que se integren al proyecto, ofreciéndoles así una oportunidad de sentirse 
dueños de un proyecto que beneficiará a sus familias y la comunidad en general.

Si el proceso se lleva a cabo, entonces el beneficio será obtener una fuente de empleo formal, que aumentará los ingresos que se requerían en la comunidad a través de la formación de una empresa que funciona a modo de cooperativa en la industria del reciclaje como se muestra en la Figura 3.

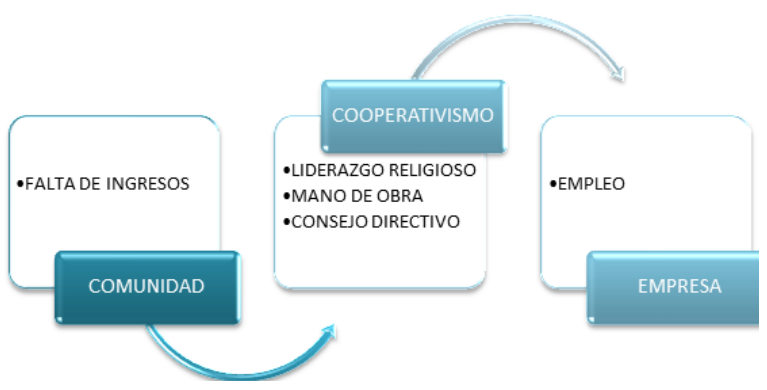

Figura 3. Proceso cooperativista

Una vez que hayan satisfecho sus necesidades básicas los socios tendrán nuevas necesidades que según la piramide de Maslow (1954) suben de nivel y ahora buscarán tener bienes y servicios a los cuales no accedían antes, por ejemplo querrán tener su propia casa o necesitarán fuentes de empleo para sus hijos. En este momento cambiarían sus motivaciones para mantenerse asociados, generando un nuevo proceso que requiere un nuevo tipo de liderazgo, recursos y forma de gobierno para brindar un nuevo beneficio a esta comunidad.

MODELO DE DESARROLLO COOPERATIVISTA EN EPS El proceso cooperativista se ejemplifica usando cinco factores que convierten las motivaciones de una comunidad en beneficios, a través de un modelo de desarrollo empresarial. Esto se explica en la Figura 4.

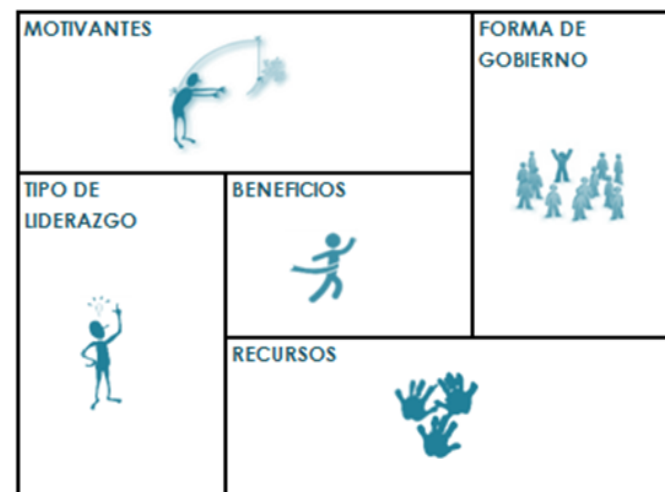

Figura 4. Modelo de desarrollo
Esta concepción permite a todas aquellas personas que intentan ayudar a una comunidad a través de un proceso cooperativista y a las cooperativas ya formadas a evaluar su situación actual y plantear estrategias de crecimiento teniendo en cuenta que el centro del proyecto es generar el beneficio requerido por los socios.

La lógica de la figura, requiere definir en primer lugar la motivación que logrará unir a los miembros de una comunidad en este proyecto. Luego se deberá elegir un tipo de liderazgo que motive a los miembros de esta comunidad a trabajar en equipo. También se deben delimitar los recursos con los que el proyecto contará y la forma de gobierno adecuada para gestionar los recursos disponibles que conlleven a generar el beneficio esperado por la comunidad, según se muestra en la Figura 5.

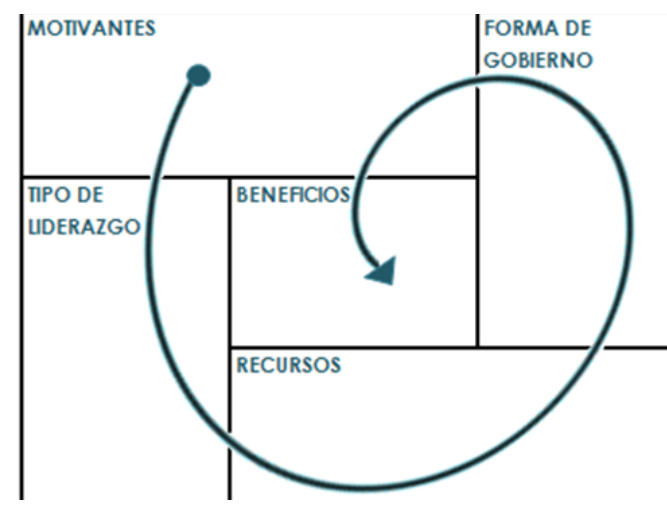

Figura 5. Uso del modelo

Esta figura se organiza considerando los dos elementos más importantes del concepto de cooperativismo: la comunidad y la empresa (Ver Figura 6). En la parte superior son mostrados los factores que afectan directamente a la comunidad, es decir, las motivantes para asociarse, el tipo de liderazgo, la forma de gobierno y los beneficios a obtener. En la parte inferior se organizan los factores que afectan a la construcción de un modelo empresarial: los recursos, la forma de gobierno y los beneficios que esta empresa debe otorgar a sus accionistas (socios).

Además, el modelo puede ser usado varias veces por una misma cooperativa de acuerdo al entorno y la situación que la misma afronte; permitiendo describir de forma dinámica su situación y estrategias más adecuadas para el desarrollo efectivo de la misma. 


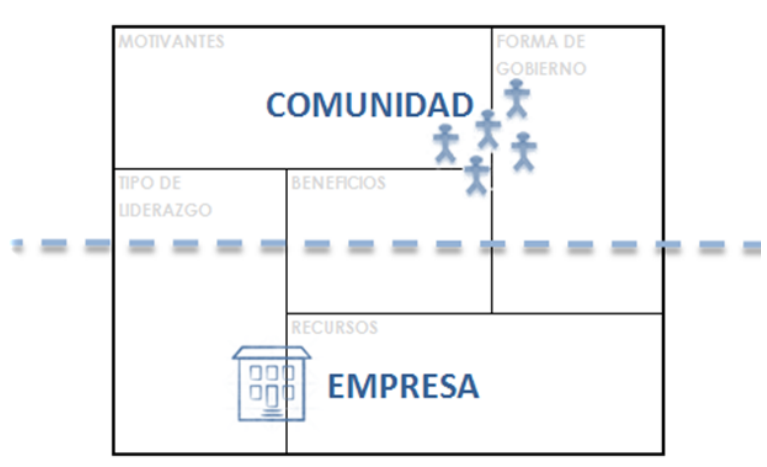

Figura 6. Elementos del modelo cooperativista

DISCUSIÓN DE LOS RESULTADOS

El cooperativismo ha demostrado su eficiencia para mejorar la calidad de vida de los segmentos de la población menos favorecidos y para conseguir beneficios mayores en las asociaciones y organizaciones de personas naturales y jurídicas. Razón por la cual, su estudio ha tomado relevancia en los últimos años. Sin embargo, el mismo se ha enfocado en estudiar el modelo para replicarlo y ayudar a otras comunidades con el patrocinio de los gobiernos y organismos internacionales.

Si bien el cooperativismo es una fórmula para ayudar a las comunidades a salir de la pobreza material e inmaterial, no es un modelo estático que puede ser copiado o replicado. El presente estudio, evidenció que este enfoque no es el adecuado, que el cooperativismo es un proceso que se debe desarrollar y que el resultado del mismo solo depende de los factores internos que intervengan en él.

El cooperativismo motiva y une a los habitantes de una comunidad que por las condiciones en las que viven (comúnmente en áreas marginadas y aisladas) no han encontrado la forma de cubrir sus necesidades. Para ello, se debe luchar contra la baja autoestima y falta de ánimos que suelen conducir al conformismo de los habitantes. Para lograrlo se requiere el arduo y desinteresado trabajo de un líder que dirija y organice a la comunidad, anclándolos en la fe y esperanza para lograr un mejor porvenir.

El caso "El Salinerito" como base del presente estudio nace del trabajo voluntario y desinteresado del padre Antonio Polo, quien sirvió de líder espiritual en los años 70' del pasado siglo para unir a los habitantes de Salinas, una comunidad olvidada de la provincia de Bolívar en Ecuador, con el único motivo de mejorar las condiciones de vida de la comunidad que estaba sumida en la miseria. La idea era clara, se debía formar una cooperativa para conseguir recursos económicos y cumplir su objetivo.

Se formó una directiva y se aprovecharon los escasos recursos que disponían sus socios. Este proceso evolucionó y hoy la comunidad es referente del desarrollo comunitario. Sin embargo, empiezan a verse problemas entre los socios y el crecimiento de las ventas se ha reducido. Esto supone que el proceso debe evolucionar hacia un nuevo modelo para mantener el crecimiento comercial y comunitario entre sus socios.

En este caso se observó que existe inconformidad entre los miembros que muestran una división clara entre las bases y los directivos a quienes denominan grandes jefes. La falta de comunicación de la misión y visión de la organización actual es la que provoca el desinterés de muchos de seguir trabajando en la cooperativa y han iniciado ya sus propios emprendimientos. Por su parte, los directivos luchan continuamente para competir comercialmente y mantener los empleos de los socios y sus familias.

El modelo planteado resume las motivantes que tiene la comunidad para asociarse, el tipo de liderazgo que forma la cooperativa, los recursos disponibles con el tipo de gobierno que administra y dirige el proceso, así como el beneficio esperado por los miembros de la comunidad. Entre las condiciones del modelo se expone que las motivantes de asociarse al inicio, son homogéneas y que las mismas van cambiando de acuerdo a las necesidades de los socios y etapas del proceso. El liderazgo es esencial para unir a los socios y hacerlos sentir que no están solos. El modelo supone que las personas siempre se motivan por la fe en algo o en alguien.

Los recursos materiales disponibles para la creación de una cooperativa normalmente son escasos, mientras que los inmateriales pueden ser abundantes. La administración de los recursos y la dirección de los socios recae sobre la directiva que se forma de acuerdo a la etapa de expansión. El gobierno debe procurar que la comunicación entre todos los socios sea efectiva y al mismo tiempo debe mantener la motivación para trabajar en conjunto y conseguir los beneficios esperados por todos.

Con este modelo se concluye que el cooperativismo como un proceso que transforma una comunidad dispersa en un modelo de desarrollo empresarial, cuyo objetivo es satisfacer las necesidades que motivan su unión. Por ello, el modelo sirve para evaluar el proceso de acuerdo a la etapa de expansión en que se encuentre. 
Esta evaluación servirá de herramienta para los socios de las cooperativas en la toma de decisiones o para elegir la mejor forma de ayudar a gobiernos y organizaciones, en general, a crear nuevas cooperativas y fortalecer el desarrollo económico de las comunidades y las naciones.

Teniendo en cuenta que el cooperativismo es una forma de desarrollo para las personas, más no una forma de administrar los negocios se debe considerar que:

1. Si se desea ayudar a una comunidad a través de un modelo de desarrollo cooperativista, el objetivo del proceso será cubrir las necesidades de sus socios y que se debe trabajar en cooperativas formando un negocio que se mueve de acuerdo a las reglas del mercado. Es decir, se debe entender que una cosa es administrar la cooperativa y sus objetivos y otra muy distinta es el modelo de gestión del negocio, el cual sigue los lineamientos del mercado en que se compite.

2. El rol del líder es fundamental, la puesta en marcha de este modelo requiere de forjar la figura de un líder que motive y genere confianza para unirse y lograr su propósito. Además, la transparencia del proceso se evidencia a través de una adecuada forma de gobierno. Por lo que, la formación de la misma es también un factor delicado de plantear.

3. Las necesidades de los socios cambian continuamente y para satisfacer las mismas los beneficios deben evaluarse continuamente. Además se debe recordar que los beneficios se cristalizan con el resultado económico, el cual depende de las condiciones de mercado y de las condiciones cooperativistas.

\section{REFERENCIAS BIBLIOGRÁFICAS}

Coraggio, J. L. (2004 a). La gente o el capital. Desarrollo local y Economía del Trabajo. Buenos Aires: Espacio Editorial.

Coraggio, J. L. (2004). Descentralizar: barajar y dar de nuevo. La participación en juego. Quito: FLACSO-Ecuador/Instituto Fronesis.

Danani, C. (2004). Política Social y Economía Social: Debates Fundamentales. Buenos Aires: Editorial ALTAMIRA.
Laville, J. L. (Coord.). (2004). Economía Social y Economía Solidaria. Una visión Europea. Buenos Aires: UNGS/ALTAMIRA/OSDE.

Maslow, A. H. (1954). Motivation and Personality. Estados Unidos de Norteamerica: Hoper \& Row.

Migliaro, L. R. (1993). Los caminos de la economía de la solidaridad. Santiago de Chile: Editorial Vivarium.

Núñez, S. (1996). La economía popular, asociativa y autogestionaria. Managua: Editorial CIPRES.

Ormaza, M. M. (2013). Desarrollo de un modelo para mejorar la calidad de vida de los recicladores informales de la ciudad de Guayaquil basado en economías populares y solidarias (Tesis dissertation). Recuperado de http://repositorio.ucsg.edu.ec/handle/123456789/1478

Osterwalder, A. y Pigneur, Y.(2012). Generación de Modelos de Negocio (5 $5^{\underline{a}}$ ed.). Barcelona: Editorial del Centro de Libros DEUSTO.

Sarria, A. y Tiribia, L. (2004). Economía Popular. Buenos Aires: Editorial Altamira.

Schüttz, G. D. Á. (2011). Límites y Posibilidades de Alianzas Progresivas en Rosario: Las relaciones políticas entre los actores de la economía social y solidaria. Buenos Aires. Congreso CIRIEC 2008.

SENPLADES. (2009 a). Plan Nacional para el Buen Vivir 20092013. Construyendo un Estado Plurinacional e Intercultural ( $1^{\underline{a}}$ ed.). Quito, Ecuador: Secretaría Nacional de Planificación y Desarrollo.

SENPLADES. (2009). Garantizar la soberanía, la paz e impulsar la integración latinoamericana. Diagnóstico SENPLADES. Quito, Ecuador. Recuperado de http://plan.senplades.gob.ec/

Superintendencia de Economía Popular y Solidaria. (23 de agosto, 2012). Ley Orgánica de la Economía Popular y Solidaria y del Sector Financiero Popular y Solidario. D.O: Registro Oficial No. 805 del 8 de octubre de 2012.

Toloza, I., Delgado, C., Cheha, C., Verdesoto, L., y Demenus, W. (2012). Enfoque territorial y modelos descentralizados de gestión del desarrollo económico Aportes desde Chile, Canadá y Ecuador. Quito, Ecuador: CONGOPE - Universidad de la Frontera (Chile).

Yunus, M. (2011). Las Empresas Sociales. Madrid: Editorial Paidos, Ibérica. 